\title{
Impact of communication delay on distributed load frequency control (dis-LFC) in multi-area power system (MAPS)
}

\author{
Auwal Mustapha Imam a, b, c, ${ }^{*}$, Kashif Chaudhary a, c, Abdullahi Bala Kunya ${ }^{\mathrm{d}}$, Zuhaib Rizvi a \\ Jalil Ali a, c \\ a Laser Centre, Ibnu Sina Institute for Scientific and Industrial Research (ISI-SIR), Universiti Teknologi Malaysia (UTM), Johor Bahru, 81300, \\ Malaysia. \\ b Physics with Electronics Department, Federal University Birnin Kebbi, 862104, Kebbi State, Nigeria. \\ c Physics Department, Faculty of Science, Universiti Teknologi Malaysia (UTM), Johor Bahru, 81300, Malaysia. \\ ${ }^{d}$ Electrical Engineering Department, Ahmadu Bello University (ABU), 1045, Zaria, Nigeria.
}

* Corresponding author: mustaphaumamauwal@gmail.com

\section{Article history}

Received 25 June 2018

Revised 29 July 2018

Accepted 7 November 2018

Published Online 25 August 2019

\begin{abstract}
In this paper, impact of communication delay on distributed load frequency control (dis-LFC) of multiarea interconnected power system (MAIPS) is investigated. Load frequency control (LFC), as one of ancillary services, is aimed at maintaining system frequency and inter-area tie-line power close to the scheduled values, by load reference set-point manipulation and consideration of the system constraints. Centralized LFC (cen-LFC) requires inherent communication bandwidth limitations, stability and computational complexity, as such, it is not a good technique for the control of large-scale and geographically wide power systems. To decrease the system dimensionality and increase performance efficiency, distributed and decentralized control techniques are adopted. In distributed LFC (dis-LFC) of MAIPS, each control area (CA) is equipped with a local controller and are made to exchange their control actions by communication with controllers in the neighboring areas. The delay in this communication can affect the performance of the LFC scheme and in a worst case deteriorates power system stability. To investigate the impact of this delay, model predictive controller (MPC) is employed in the presence of constraints and external disturbances to serve as LFC tracking control. The scheme discretizes the system and solves an on-line optimization at each time sample. The system is subjected to communication delay between the CAs, and the response to the step load perturbation with and without the delay. Time-based simulations were used on a three-area MAIPS in MATLAB/SIMULINK environment to verify the investigations. The overshoot and settling time in the results reveals deterioration of the control performance with delay. Also, the dis-LFC led to zero steady states errors for frequency deviations and enhanced the MAIPS' performance. With this achievement, MPC proved its constraints handling capability, online rolling optimization and ability to predict future behavior of systems.
\end{abstract}

Keywords: Control Area (CA), communication delay, LFC, MPC, MAIPS.

\section{INTRODUCTION}

Electrical power system is one of the largest, geographically expansive and most complex-engineered systems ever created [1] Owing to this, real power systems are segregated into several interconnected subsystems best known as control areas (CA) as shown in Fig. 1. Each of the control areas has its own group of generators, $P_{G_{i, j}}$ (mostly three phase) which are directly responsible for its exchanges of power, $P_{i j}$ and loads $P_{L_{i, j}}$ with adjacent CAs through the tie-lines [2]. Each of the subsystems exchange their predictions, information and measurement via communication, and at the same time incorporate information and coordinate with each other [3, 4]. In such multi-area interconnected power systems (MAIPS), there is scheduled power variations of the tie-line and undesired frequency due to discrepancy between the total generated power and load demands [5]. The generators may be forced out of synchronism, and eventually the system would collapse if the continous frequency deviation issues are not solved [6].

System frequency is one of the most substantial indicators of power quality, whose constancy is correlated to safety and efficacy of almost all electrical appliances [7]. To maintain system frequency and interarea tie-line power within acceptable limits, by manipulating the load reference set-points, load frequency control (LFC) is introduced. Effective tracking of MAPS load demands, maintaining zero steady states errors for frequency deviations and performance enhancement are the main aims of LFC [8]. In LFC, these problems in the dynamical operation of MAIPS are theoretically defined as large-scale disturbamce with input and state constraints [5].

Analogous to the control of many other dynamical systems, LFC is achevived in either centralized or decentralized method in MAIPS. The centralized control, also known as supervisory control is the one in which a single controller measures all the outputs of the CAs, compute the control soution optimally and apply solution to all the actuators of each of the CA of the network, within one sampling period $[2,7,9,10]$.

Conversely, in non-centralized control further classified as decentralized and distributed control, each CA is equipped with one controller which autonomously computes the optimal control solution and apply that action to the actuators in that particular CA. In the latter, there is communication between neighboring CAs, while in the former, such is missing while assuming negligible effects of the interconnection 
[10]. In many situations, however, this assumption is illogical and abridge the performance of the whole control [11]. There is need for some communication levels between various controllers in a distributed patter in order to better closed-loop performance of the control.

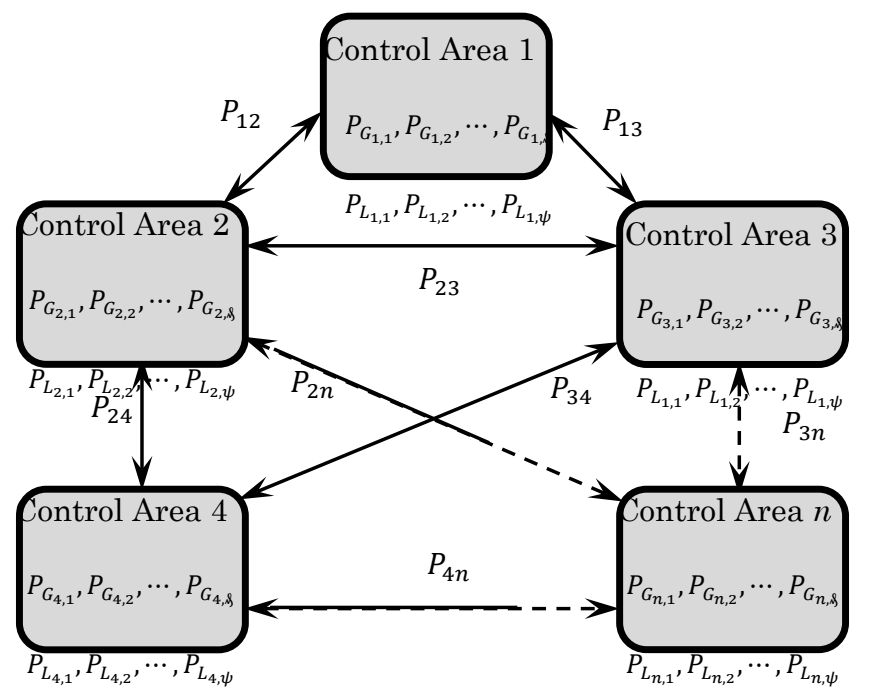

Fig. 1 MAIPS schematics.

Previously, intelligent systems applications have received impressive attention in different areas of power systems such as planning, operations, management and control [12]. Numerous research publications [13, 14] have indicated the applications of intelligent systems to power systems or even control methodologies using fuzzy logic [15] and other intelligent controllers. Numerous control strategies have been proposed by several researchers in order to get an enhanced control performance. Shiroei et al. [16] developed a hierarchical twolevel LFC. Standard proportional-integral (PI) controllers were applied at the lower level, while MPC was applied at the higher level as a supervisory controller to ascertain the optimal setpoint in the lower level for the PI controllers. Centralized model predictive controller (c-MPC) was applied in [17] and model predictive load frequency control in [18]. In all these research works together with many others, centralized MPC is employed.

Therefore, as power networks are large-scale systems, both computationally and geographically, a cen-MPC controller is practically impossible to implement in such systems. However, the rapid expansion and rise in dimensions of modern power systems is gradually rendering the centralized LFC scheme worthless, paving way for distributed and decentralized control techniques to remarkably decrease the dimensions of the systems $[19,20]$.

Dis-LFC strategy is viewed as networked control system (NCS), from which the basic priciple of smart power grid originates. It interconnects controllers and actuators on a communication network for data exchange during control and monitoring of geographically spread generators in the power system towards realization of smart grid. [2123]. Even though, the stability and robustness of dis-LFC depends mainly on the communication infrastructure performance, many studies on this subject ignore the delay caused by the transmission of data via communication channels between CAs, like in [21, 24, 25].

In this study, impact of communication delay on MPC based disLFC of three-area MAIPS is investigated. The choice of this controller is based on its constraints handling capability, online rolling optimization and ability to predict future behavior of the system. Control input limits, frequency deviation limits, terminal equality, governor dead band (GDB) and generation rate contraints (GRC) are considered as constraints. Comparison is made between the response of the system with cen-LFC, dis-LFC and delayed dis-LFC scheme.

The dynamics of the interconnected of MAIPS are descibed by mathematical modeling as presented in Section II of this paper. Systematic design of the centralized and distributed MPC-LFC and incorporated communication delay is presented in Section III. Tests illustrations, results obtained and discussion of the results are presented in section IV while Section V carries conclusion.

\section{DYNAMICS OF MAIPS}

Notwithstanding the complexity of power system dynamics, let's consider a MAIPS with $n$ CAs as illustrated in Fig. 1. Each area has $\&$ number of generators and $\psi$ load points. The states of the system include the deviation of frequency, tie-line power, gas valve positioning and mechanical output of each generator. While the load perturbation is the input to the system with area control error (ACE) as the output.

In each CA, deviation of frequency from its rated value indicates interconnection power mismatch and generation-demand balance in the CA. For a given CA, $i$ the effect of this frequency mismatch is modelled in a linearized swing equation illustrated in Eqn. (1).

$$
\dot{f}_{i}=\frac{1}{H_{i}}\left(P_{G_{i}}-D_{i} f_{i}-P_{i}^{t i e}-P_{D_{i}}\right)
$$

Where $f_{i}$ is the deviation of the $i$ th area frequency. Based on the logical practical assumption that all generators in a CA form a coherent group (they all swing in unison) [26], single frequency is assumed for each CA.

$$
P_{G_{i}}=\sum_{j=1}^{\&} P_{G_{i, j}} ; \quad \forall \text { Generators }
$$

$$
P_{D_{i}}=\sum_{j=1}^{\Re} P_{D_{i, j}} ; \quad \forall \text { Loads }
$$

Using approximated DC power flow modelling, the power flow between connected CAs is expressed as,

$$
\dot{P}_{i}^{t i e}=\sum_{j \in \mathcal{A}_{i}^{t i e}} \dot{P}_{i j} ; \quad \dot{P}_{i j}=2 \pi T_{i j}^{\hat{o}}\left(f_{i}-f_{j}\right)
$$

The dynamics of the employed non-reheat turbines depends on the control actions initiated by the supplementary LFC and power deviations [5]. The turbine dynamics of the $i$ th CA for small deviations is modelled as: -

$$
\dot{P}_{G_{i, \S}}=\operatorname{sat}_{\dot{P}_{i, \$}^{G}}^{i}\left\{\frac{1}{T_{T_{i, \$}}}\left(P_{i, \$}^{G o v}-P_{i, \oint}^{G}\right)\right\}
$$

Table 1 provides the definitions of the terms used in the formulation.

The change in the position of governor valve related to frequency for the sth generator in $i$ th CA is simplified so it can have single-time constant, $T_{\text {Gov }_{i, \AA}}[\mathrm{s}]$, modelled as;

$$
\dot{P}_{i, \S}^{G o v}=\frac{1}{T_{G o v}}\left(P_{i, \$}^{S C}-P_{i, \$}^{G o v}-\frac{1}{R_{i, \$}} f_{i}\right)
$$

ACE is used as control reference and performance measure in LFC. It is obtained by combinations of tie-line power and frequency deviations. Thus, for $i$ th CA,

$$
\operatorname{ACE}_{i}=\beta_{i} f_{i}+P_{i}^{t i e}
$$

The continuous LFC time model in MAIPS described; (1) - (7) for $i$ th CA can be summarized in state space model; 
Table 1 System symbols.

\begin{tabular}{|c|c|}
\hline Symbol & Description \\
\hline$\tau_{d_{i}}$ & Time delay in area $i[\mathrm{~s}]$. \\
\hline$\overline{f_{i}}$ & Frequency deviation of ith $\mathrm{CA}[\mathrm{Hz}]$. \\
\hline$P_{G_{i, 8}}$ & Generator power output change in s in the ith CA [pu MW]. \\
\hline$P_{i}^{t i e}$ & Net tie-line power deviation in ith CA [pu MW]. \\
\hline$D_{i}$ & ith CA damping coefficient equivalent in [pu MW/Hz]. \\
\hline$P_{D_{i}}$ & ith CA total load demand change [pu MW]. \\
\hline$T_{i j}^{\hat{0}}$ & $\begin{array}{l}\text { Coefficient of synchronization between area } i \text { and } j[\mathrm{pu} \\
\mathrm{MW} / \mathrm{Hz}] \text {. }\end{array}$ \\
\hline$\overline{H_{i}}$ & Inertia constant equivalent [pu s]. \\
\hline$\overline{\beta_{i}}$ & ith CA scalar frequency bias parameter [pu MW/Hz] \\
\hline$P_{i, \$}^{G \text { Gov }}$ & ith CA generator s governor team valve position \\
\hline$R_{i, \uparrow}$ & ith CA generator \& droop characteristics [pu MW/Hz]. \\
\hline 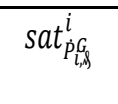 & $\begin{array}{l}\text { CA i generator \& saturation nonlinearity for GRC } \\
\text { modelling. }\end{array}$ \\
\hline$T_{\text {Govi }_{i, \AA}}$ & ith CA generator \& governor time constant. \\
\hline$T_{T_{i, \Re}}$ & ith CA generator \& steam chest time constant. \\
\hline$N_{p}$ & Horizon of MPC finite prediction \\
\hline $\mathrm{ACE}_{i}$ & th CA area control error. \\
\hline
\end{tabular}

The change in the position of governor valve related to frequency for the $s$ th generator in $i$ th CA is simplified so it can have single-time constant, $T_{\text {Gov }_{i, \Omega}}[\mathrm{s}]$, modelled as;

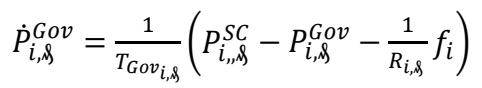

ACE is used as control reference and performance measure in LFC. It is obtained by combinations of tie-line power and frequency deviations. Thus, for $i$ th CA,

$$
\mathrm{ACE}_{i}=\beta_{i} f_{i}+P_{i}^{\text {tie }}
$$

The continuous LFC time model in MAIPS described; (1) - (7) for $i$ th CA can be summarized in state space model;

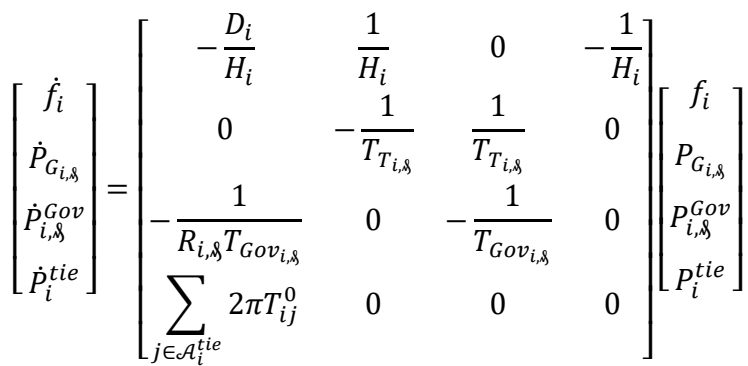

$$
\begin{aligned}
& +\left[\begin{array}{c}
0 \\
0 \\
\frac{1}{T_{\text {Gov }_{i, \$}}} \\
0
\end{array}\right] P_{i, \S}^{S C}+\left[\begin{array}{c}
-\frac{1}{H_{i}} \\
0 \\
0 \\
0
\end{array}\right] P_{i}^{D}+\left[\begin{array}{c}
-\frac{1}{H_{i}} \\
0 \\
0 \\
-2 \pi \sum_{i, j} T_{i j}^{0}
\end{array}\right] f_{j} \\
& \mathrm{ACE}_{i}=\left(\begin{array}{llll}
\beta_{i} & 0 & 0 & 1
\end{array}\right)\left[\begin{array}{c}
f_{i} \\
P_{G_{i, \S}} \\
P_{i, \AA}^{G o v} \\
P_{i}^{t i e}
\end{array}\right]
\end{aligned}
$$

where $\left[\begin{array}{llll}f_{i} & P_{i, \S}^{G} & P_{i, \S}^{G o v} & P_{i}^{t i e}\end{array}\right]^{T} \in \mathbb{R}^{4}=$ state vector,

$\left(x_{i}\right), P_{i, \S}^{S C}=$ control input $\left(u_{i}\right)$,

$P_{i}^{D}=$ disturbance input and,

$\mathrm{ACE}_{i}$ is considered as the output for $i$ th CA.

Saturation within a typical value of $\pm 10 \% / \mathrm{min}$ is used to model the generator GRC constraint. The reference setpoint is constrained as in,

$$
\left|P_{i, \Omega}^{S C}\right| \leq 0.3 p u M W
$$

By considering the delay in the delivery of the state parameters, the ACE will be remodeled as;

$$
\operatorname{ACE}_{i}(t)=\beta_{i} f_{i}\left(t-\tau_{d_{i}}\right)+P_{i}^{t i e}\left(t-\tau_{d_{i}}\right)
$$

\section{DISTRIBUTED MODEL PREDICTIVE CONTROL (d-MPC)}

In MAIPS, two different control loops known as primary and supplementary speed loops are used to accomplish LFC [27]. Supplementary control loop of area has an MPC controller [5].

MPC is a control technique for solving online optimal control issues by consideration of system dynamics and its output constraints. The main motivation of MPC is to derive a system model at a particular operating point on which the output signals are expressed in terms of future and past control signals, and at the same time performing forecast optimization based on some pre-specified criterion [28]. MPC has been extensively applied in industries to effectively solve multivariable constrained control problems [16]. Fig. 2 shows the Simulink simulation model of the three-area MAIPS dynamic. The model was used to generate the response when the MPC controller was applied.

MPC make use of an explicit system model that predict future trajectory of system outputs. The difference between the predicted ACE and reference output is known as the prediction error. The actions of control inputs are reduced over a future horizon, subject to manipulated inputs, outputs and states constraints [3]. To minimize the mismatch and future disturbance effect, the first control signal in the optimal sequence is the only signal applied on the actuator [29]. With the state vector, $x_{i}$, 
the subsystem $i$ partitioned model of the three-area power system is described in state-space model as,

$$
\begin{array}{r}
\dot{x}_{i}(t)=A_{i i} x_{i}(t)+B_{i i} u_{i}(t)+D_{i i} w_{i}(t)+\sum_{j \neq i} A_{i j} x_{i}(t)+B_{i j} u_{j}(t) \\
A_{i i}=\left[\begin{array}{cccc}
-\frac{D_{i}}{H_{i}} & \frac{1}{H_{i}} & 0 & -\frac{1}{H_{i}} \\
0 & -\frac{1}{T_{T_{i, S}}} & \frac{1}{T_{T_{i, \$}}} & 0 \\
-\frac{1}{R_{i, \$} T_{G o i_{i, s}}} & 0 & -\frac{1}{T_{G o v_{i, \$}}} & 0 \\
\sum_{j, i}^{2 \pi T_{i j}^{0}} & 0 & 0 & 0
\end{array}\right], D_{i i}=\left[\begin{array}{c}
-\frac{1}{H_{i}} \\
0 \\
0 \\
0
\end{array}\right]
\end{array}
$$

$$
A_{i j}=\left[\begin{array}{cccc}
0 & 0 & 0 & 0 \\
0 & 0 & 0 & 0 \\
0 & 0 & 0 & 0 \\
-\sum_{j} 2 \pi T_{i j}^{0} & 0 & 0 & 0
\end{array}\right], B_{i j}=[0]_{4 \times 1}
$$

In this study, equal control and prediction horizons are chosen. The system model can either be represented in state-space or transfer function. In this study, the plant is represented in state space format as shown in (9) and (13). Each MPC controller solves a local optimization problem [10], while exchanging the information with other controllers. Time delay is created between the controllers.

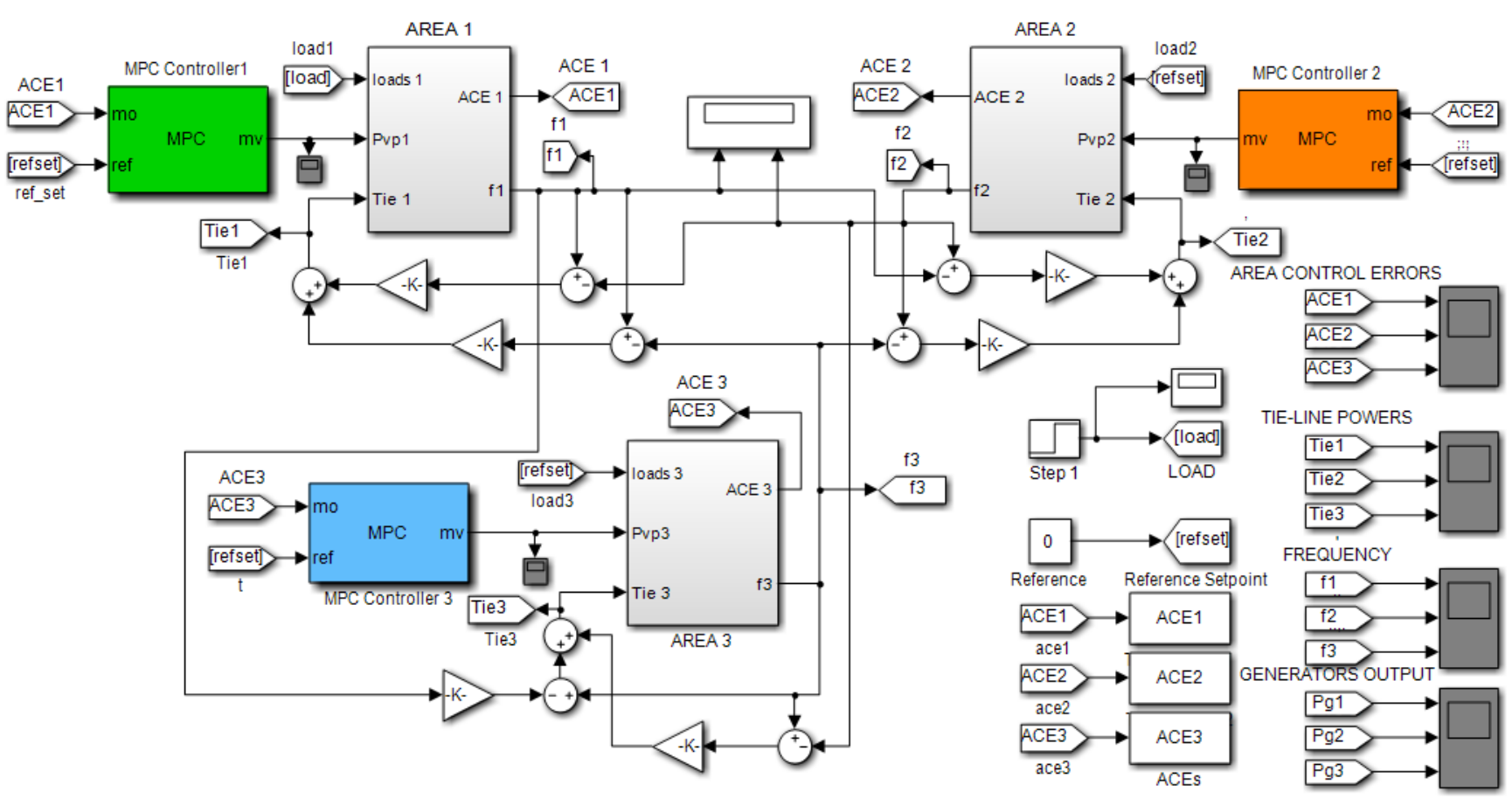

Fig. 2 Three-area MAIPS dynamic simulation Simulink model on.

\section{MPC-based LFC}

Fig. 2 illustrates the design of dis-MPC for three-area interconnected MAIPS LFC. Each CA is equipped with a local controller and are made to exchange their control actions by communication with controllers in the neighboring areas. The control actions are then applied to the actuators of the system. In this regard, the actuators actually the valve of the gas or water inlet (in the case of hydro power plant). The delay in this communication can affect the performance of the LFC scheme and in a worst case deteriorates power system stability. The continous time system of MPC is discretized because its optimization is done for certain time sampling. The optimization problem for each CA $i$ can be;

$$
\min _{u_{i}(*)} J_{i}\left\{x_{i}(t), \bar{u}_{i}(*)\right\}
$$

So that

$$
\begin{aligned}
& J_{i}\left\{x_{i}(t), \tilde{u}_{i}(*)\right\}= \\
& \quad \int_{t}^{t+N_{p}}\left(\left\|\tilde{x}_{i}\left(\kappa ; x(t), t-x_{i d}\right)\right\|_{M_{i}}^{2}+\left\|u_{i}(\kappa)\right\|_{N_{i}}^{2}\right) d \kappa
\end{aligned}
$$

Subject to (8) and (9) discritization as constraints, where the arbtrary dicrete time control horizon sample is $\kappa \in\left[t, t+N_{P}\right]$, the positive symmetric and definite weighting matrices employed as tuning parameters, which are chosen arbitrarily to achive the desired control performance are $M_{i}$ and $N_{i}$. The subsystem (7) predicted trajectory which start from actual state $x_{i}(t)$ at time $t$ is $\tilde{x}_{i}(\kappa ; x(t), t)$. 
The summarized dis-MPC algorithm as used here is,

\section{Step 1: Information Propagation}

$i$ th $\mathrm{CA}$ communicate out its previous predictions $\tilde{x}_{i}\left(\kappa ; x\left(t_{\mu-1}\right), t_{\mu-1}\right), \kappa \in\left[t_{\mu}, t_{\mu-1}+N_{P}\right]$ to other controllers and also receive information $\tilde{x}_{j}\left(\kappa ; x\left(t_{\mu-1}\right), t_{\mu-1}\right), \kappa \in\left[t, t_{\mu-1}+N_{P}\right]$ from them.

\section{Step 2: Initialization}

The given measured predicted trajectory, $\tilde{x}_{i}(t)$, set $\tilde{x}_{i}(t)=x_{i}(t)$ at each sampling time $t_{\mu}$,

\section{Step 3: Optimization}

The rolling optimal control problem in (10) is solved using quadratic programming.

\section{Step 4: Assignment}

Assign $u_{i}\left(t_{\mu}\right)=\tilde{u}_{i}\left(t_{\mu}\right)$ or $u_{i}\left(t_{\mu}\right)=\tilde{u}_{i}\left(t_{\mu-1}\right)$ if the optimal control problem (12) in step 3 is feasible.

\section{Step 5: Estimation/Prediction}

Predict the future states $\tilde{x}_{i}\left(\kappa ; x\left(t_{\mu}\right), t_{\mu}\right), \kappa \in\left[t_{\mu+1}, t_{\mu}+N_{P}\right]$ with the optimized control sequence.

\section{Step 6: Implementation/Actuation}

Apply the optimal control sequence $u_{i}\left(t_{\mu}\right)$ to the actuator, set $\mu=$ $\mu+1$. Return step 1 at the next sample time.

\section{RESULTS AND DISCUSSION}

A model predictive controller (MPC) posing the load frequency control (LFC) is emplyed problem in the presence of external disturbances and constraints as a tracking control problem to investigate the impact of communication delay. The system is subjected to communication delay between the CAs (with single generator each), and the response to the step load perturbation with and without the delay is studied.

In this test, only CA 1 is subjected to $10 \%$ load increment, while in the other two CAs are concurrently left unperturbed. The load disturbance rejection performance of the dis-MPC formulation is evaluated and compared with the same scenario but delayed from $1 \mathrm{sec}$.

The system's responses are illustrated in Fig. $3-5$. It clearly shows that at the steady state, the tie-line power deviation settles at their expected values and the local frequency deviation of each CA converges to zero. Thus, the impact of the time delay on the frequency deviations $\left(f_{1}, f_{2}, f_{3}\right)$, ACEs and tie-line powers can be ascertain using overshoot and settling time. Table 2 summarizes the overshoot and settling of the system states with and without the delay.

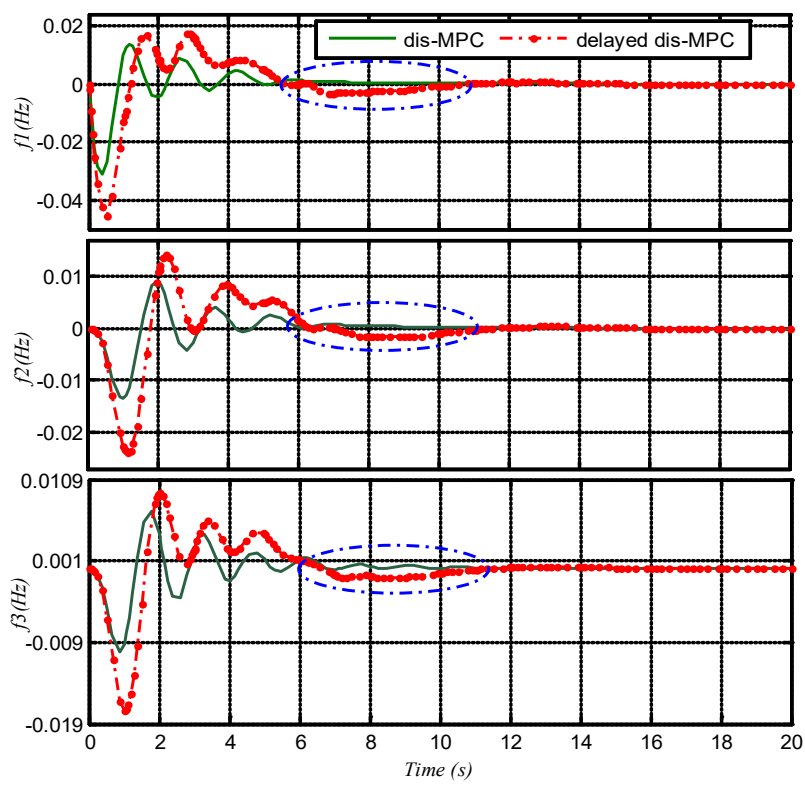

Fig. 3 Frequency deviations with and without 1.0 sec delay.

Table 2 Overrshoot and settling time of the states.

\begin{tabular}{|c|c|c|}
\hline States & $\begin{array}{l}\text { Maximum Overshoot } \\
(\mathrm{pu})\end{array}$ & Settling time (s) \\
\hline \multicolumn{3}{|l|}{ Without delay } \\
\hline${ }_{1}, f_{2}, f_{3}$ & $-0.031,-0.013,-0.010$ & $8.46,8.46,9.49$ \\
\hline$P_{1}^{\text {tie }}, P_{2}^{\text {tie }}, P_{3}^{\text {tie }}$ & $-0.022,0.012,0.01$ & $7.70,8.57,8.47$ \\
\hline $\begin{array}{l}A C E_{1}, A C E_{2}, \\
A C E_{3}\end{array}$ & $0.021,0.00374,0.0029$ & $6.17,7.193,7.13$ \\
\hline$P_{G_{1}}, P_{G_{2}}, P_{G_{3}}$ & $0.106,0.022,0.016$ & $7.51,7.45,8.73$ \\
\hline \multicolumn{3}{|c|}{ With 1.0 sec delay } \\
\hline $1, f_{2}, f_{3}$ & $-0.042,-0.023,-0.16$ & $11.50,10.79,10.52$ \\
\hline$P_{1}^{t i e}, P_{2}^{t i e}, P_{3}^{t i e}$ & $-0.035,-0.015,-0.012$ & $11.18,9.49,10.06$ \\
\hline $\begin{array}{l}A C E_{1}, A C E_{2} \\
A C E_{3}\end{array}$ & $0.034,0.0043,0.0038$ & $13.17,10.06,8.57$ \\
\hline$P_{G_{1}}, P_{G_{2}}, P_{G_{3}}$ & $0.12,0.04,0.028$ & $16.54,16.31,16.86$ \\
\hline
\end{tabular}




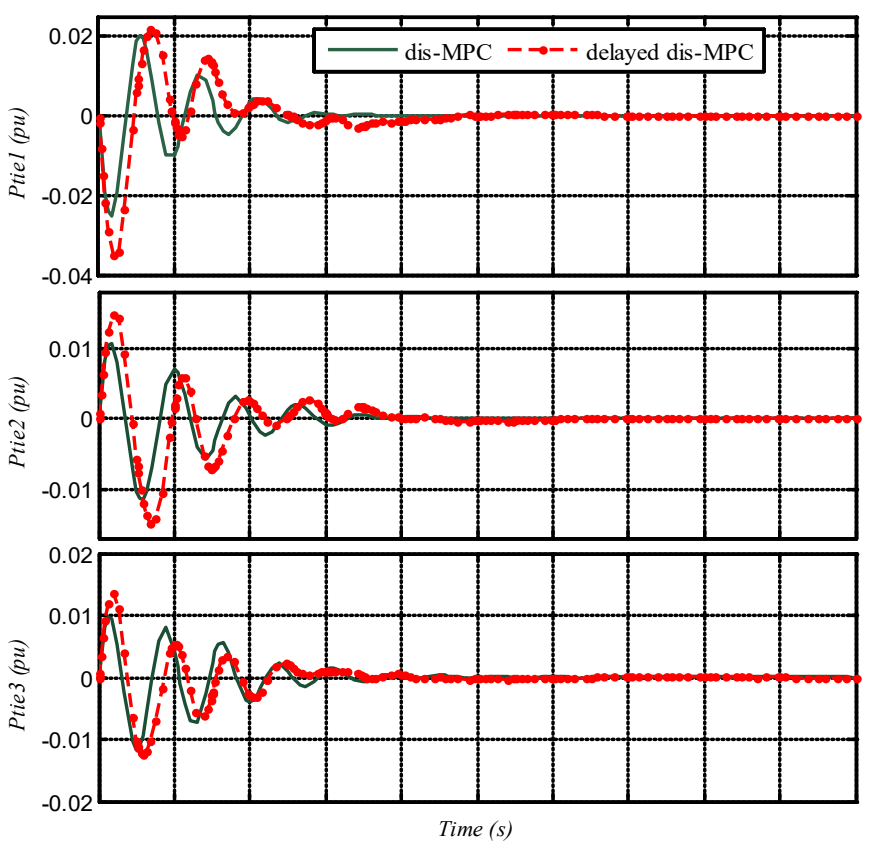

Fig. 5 Tie-line power deviations with and without $1.0 \mathrm{sec}$ delay.

It can be inferred from Fig. 5 that power flows from CA 2 and 3 to CA 1 (negative overshoot) where load change occurred. However, at steady state all the tie-line powers are forced to zero.

In order to preserve zero tie-line powers, generator(s) each CA should provide for its load demands at steady state. This assertion is observed in the response of the generators in CA 1-3 illustrated in Fig. 5. Since only CA 1 is perturbed, the generator in CA 1 generates the $10 \%$ pu $M W$ at steady, while the rest of the generators settle at zero. Thus, at steady state;

$$
P_{1}^{G}=0.1 p u M W, P_{2}^{G}=0.0 p u M W, P_{3}^{G}=0.0 p u M W
$$
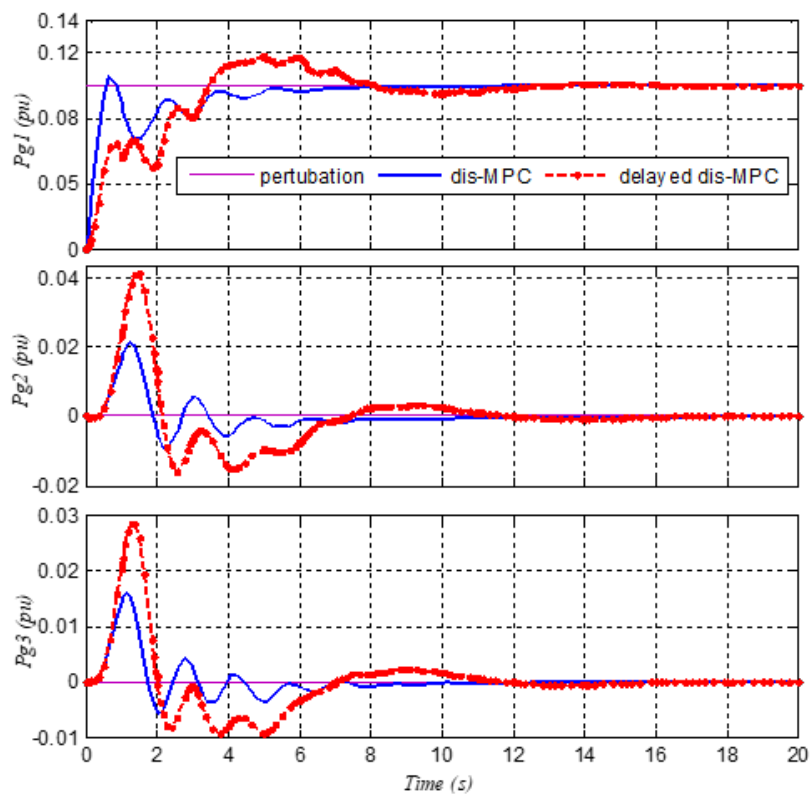

Fig. 4 Deviation in output power of the generators with and without $1.0 \mathrm{sec}$

It is imperative to point out that there are many factors which affect the dis-LFC performance apart from time delay [10]. The MPC variables estimation and weight tuning, control interval, predictions and other factors are among the factors that affect the performance of MPC rolling and optimization-based control [5].
It is envisioned in the future work to adapt the time delay in the controller design so as reject its effect and that of the perturbation concurrently. This is feasible taking the predictive nature of the MPC into cognizance.

\section{CONCLUSION}

Impact of time delay on dis-MPC is investigated in this paper. The dis-MPC is designed as a tracking control problem in the presence of external disturbances and constraints. The MAIPS dynamics are studied. The control areas that constitute the MAIPS is equipped with one MPC controller and are made to exchange their predictions and measurements through communication and incorporate other information from various controllers into their local control scheme. The optimized input control sequence generated by the MPCs is used to adjust the CAs thermal generator valve position in the. Delay in this communication pose a threat to the stability of the LFC. Three-area MAIPS time-based simulations are used to verify the investigations. The system is subjected to communication delay between the CAs (with single generator each), and the response to the step load perturbation with and without the delay is studied. In this test, only CA 1 is subjected to $10 \%$ load increment, while in the other two CAs are concurrently left unperturbed. It can be inferred from the simulation that time delay of $1.0 \mathrm{sec}$ has deteriorates the frequency stability by almost $34.38 \%$ in the affect area while the tie-line power by $34.24 \%$.

\section{AKNOWLEDGEMENT}

The authors would like to thank Ibnu Sina Institute for Scientific and Industrial Research (ISI-SIR), Universiti Teknologi Malaysia (UTM) for providing research facilities and UTM for providing support through IDF. This research work has supported by Tier 1 (16H42) Grant.

\section{REFERENCES}

[1] Khalil, A., Rajab, Z., Alfergani, A., Mohamed, O. 2017. The impact of the time delay on the load frequency control system in microgrid with plug-inelectric vehicles. Sustainable Cities and Society, 35, 365-377.

[2] Wood, A. J., Wollenberg, B. F. 2012. Power generation, operation, and control. John Wiley \& Sons.

[3] Ma, M., Chen, H., Liu, X., Allgower, F. 2014. Distributed model predictive load frequency control of multi-area interconnected power system. International Journal of Electrical Power \& Energy Systems, 62, 289-298.

[4] Imam, A. M., Tampul, H. M., Bako, A., Mohammed, J. 2016. Design of a scada-based water flow and distribution monitoring system. African Journal of Science and Research, 5(2), 56-59.

[5] Kunya, A., Argin, M. 2018. Model predictive load frequency control of multi-area interconnected power system. 2018 IEEE Texas Power and Energy Conference (TPEC). 8-9 Feb, USA.

[6] Bevrani, H. 2009. Robust Power System Frequency Control. Springer.

[7] Sekhar, G. T. C., Sahu, R. K., Baliarsngh, A. K., Panda, S. 2016. Load frequency control of power system under deregulated environment using optimal firefly algorithm. International Journal of Electrical Power \& Energy Systems, 74, 195-211.

[8] Shayeghi, H., Shayanfar, H., Jalil, A. 2009. Load frequency control strategies: A state-of-the-art survey for the researcher. Energy Conversion and mManagement, 50(2), 344-353.

[9] Ejegi, E., Rossiter, J., Trodden, P. 2016. Distributed model predictive load frequency control of a deregulated power system. 2016 UKACC 11th International Conference on Control (CONTROL). 31 Aug.-2 Sept, United Kingdom.

[10] Ma, M., Zhang, C., Liu, X., Chen, H. 2017. Distributed model predictive load frequency control of the multi-area power system after deregulation. IEEE Transactions on Industrial Electronics, IEEE Transactions on Industrial Electronics, 64(6), 5129-5139.

[11] Shree, S. B., Kamaraj, N. 2016. Hybrid neuro fuzzy approach for automatic generation control in restructured power system. International Journal of Electrical Power \& Energy Systems, 74, 274-285.

[12] Bevrani, H., T. Hiyama. 2016. Intelligent Automatic Generation Control. CRC Press.

[13] Vale, Z., Venayagamoorthy, G. K., Ferreira, J., Morais, H. 2011. Computational intelligence applications for future power systems. Computational Intelligence for Engineering Systems, 176-193. 
[14] Fozdar, M., C. Arora, V. Gottipati. 2007. Recent trends in intelligent techniques to power systems. 2007 42nd International Universities Power Engineering Conference. 4-6 Sept, United Kingdom.

[15] Alata, M., Al-Nimr, M., Qaroush, Y. 2005. Developing a multipurpose sun tracking system using fuzzy control. Energy Conversion and Management, 46(7), 1229-1245.

[16] Shiroei, M., Ranjbar, A. 2014. Supervisory predictive control of power system load frequency control. International Journal of Electrical Power \& Energy Systems, 61, 70-80.

[17] Fu, D., Ionescu, C. M., Aghezzaf, E-H, Keyser, R. D. 2014. Decentralized and centralized model predictive control to reduce the bullwhip effect in supply chain management. Computers \& Industrial Engineering, 73, 2131.

[18] Ersdal, A. M., Imsland, L., Uhlen, K. 2016. Model predictive loadfrequency control. IEEE Transactions on Power Systems, 31(1), 777-785.

[19] Sagar, P. S. V., Swarup, K. S. 2016. Load frequency control in isolated micro-grids using centralized model predictive control. IEEE Access, 16241-16251.

[20] Zhang, Y., Liu, X., Qu, B. 2017. Distributed model predictive load frequency control of multi-area power system with DFIGs. Journal of Automatica Sinica, 4(1), 125-135.

[21] Shiroei, M., Toulabi, M. R., Ranjbar, A. M. 2013. Robust multivariable predictive based load frequency control considering generation rate constraint. International Journal of Electrical Power \& Energy Systems, 46, 405-413.

[22] Rakhshani, E., Sadeh, J. 2010. Practical viewpoints on load frequency control problem in a deregulated power system. Energy Conversion and Management, 51(6), 1148-1156.

[23] Bensenouci, A., Ghany, A.A. 2010. Performance analysis and comparative study of LMI-based iterative PID load-frequency controllers of a singlearea power system. WSEAS Transactions on Power Systems, 2(5), 85-97.

[24] Tan, W., Hao, Y., Li, D. 2015. Load frequency control in deregulated environments via active disturbance rejection. International Journal of Electrical Power \& Energy Systems, 66, 166-177.

[25] Zheng, Y., Zhou, J., Xu, Y., Zhang, Y., Qian, Z. 2017. A distributed model predictive control based load frequency control scheme for multi-area interconnected power system using discrete-time Laguerre functions. ISA transactions, 68, 127-140.

[26] Yazdizadeh, A., Ramezani, M. H., Hamedrahmat, E. 2012. Decentralized load frequency control using a new robust optimal MISO PID controller. International Journal of Electrical Power \& Energy Systems, 35(1), 57-65.

[27] Elsisi, M., Soliman, M., Aboelela, M. A. S., Mansour, W. 2016. Ba inspired algorithm based optimal design of model predictive load frequency control. International Journal of Electrical Power \& Energy Systems, 83, 426-433.

[28] Kumtepeli, V., Wang, Y., Tripathi, A. 2016. in Multi-area model predictive load frequency control: A decentralized approach. 2016 Asian Conference on Energy, Power and Transportation Electrification (ACEPT). 25-27 Oct, Singapore.

[29] Shi, X.,Hu, J., Yu, J., Yong, T., Cao, J. 2015. A novel load frequency control strategy based on model predictive control. 2015 IEEE Power \& Energy Society General Meeting. 26-30 July, United States. 